\title{
Exacerbación de asma en una clínica de IV nivel en Barranquilla: características clínicas y sociodemográficas
}

\author{
Asthma exacerbation in an IV level \\ clinical at Barranquilla: clinical and \\ sociodemographic characteristics
}

\begin{abstract}
Mary Luz Moreno-Vega ${ }^{1}$, Jennifer Paola Santiago-Barrios ${ }^{1}$, ERnesto Santiago ${ }^{2}$, Lourdes Varela-Prieto ${ }^{3}$, Laura Alejandra Mora ${ }^{4}$, Soraya Salcedo ${ }^{4}$.
\end{abstract}

\section{Resumen}

INTRODUCCIÓN: una fracción substancial de pacientes con diagnóstico de asma pertenecen al subgrupo de "no controlados" y tienen un mayor riesgo de presentar exacerbaciones, que es la principal causa de morbilidad y mortalidad en el asma, además de ser el motivo principal para acudir a los servicios de urgencias. Los datos disponibles sobre hospitalizaciones e incidencia de la exacerbación asmática en urgencias proceden de países anglosajones. En Colombia existen datos de subdiagnóstico de la enfermedad y un aumento en la prevalencia, pero faltan estudios que evalúen las características epidemiológicas de los pacientes adultos con exacerbación asmática.

MATERIAL Y MÉTODOS: estudio observacional, descriptivo, retrospectivo, de corte transversal, que incluyó 523 historias clínicas de pacientes con exacerbación asmática atendidos en la sala de urgencias de un centro de salud de IV nivel en Barranquilla, entre enero y diciembre de 2017.

RESULTADOS: el 0,93\% del total de urgencias atendidas durante 2017 correspondieron a pacientes con exacerbación asmática, mayores de 16 años. La media de exacerbaciones atendida fue de 1,43 al día; la edad media fue de $47 \pm 19$ años; el $68 \%$ eran mujeres; el 13,1 $\%$ de los eventos se presentaron en el mes de septiembre, y el principal factor detonante asociado fue la infección viral en el 93,2\%.

CONCLUSIONES: los datos de este estudio destacan la importante carga asistencial que representan las exacerbaciones asmáticas en una población adulta, como motivo de atención en los servicios de urgencias y con mayor frecuencia en la época de lluvia, y sugiere que las infecciones respiratorias virales podrían ser la causa subyacente.

Palabras clave: asma, exacerbación, clínica, sociodemográfico.

\begin{abstract}
INTRODUCTION: a substantial fraction diagnosed patients with asthma belong to "uncontrolled" subgroup and have higher risk to present exacerbations, being morbidity and mortality cause main; besides being reason main to go at the emergency department. Available hospitalizations data and asthma exacerbation incidence at the emergency department come Anglo-Saxon countries. At Colombia, there are underdiagnosis disease and an increased prevalence, but there are not studies evaluating epidemiological characteristics adult patients with asthma exacerbations.
\end{abstract}

${ }^{1}$ Residente de tercer año, especialización en Medicina Interna, Universidad Libre, seccional Barranquilla.

${ }^{2}$ Internista neumólogo y docente adscrito de posgrados, Universidad Libre, seccional Barranquilla. Organización Clínica General del Norte. Barranquilla, Colombia.

${ }^{3}$ Docente de investigación adscrita a posgrados, Universidad Libre Seccional Barranquilla.

${ }^{4}$ Epidemióloga clínica, Organización Clínica General del Norte. Barranquilla, Colombia.

Autor de correspondencia

Mary Luz Moreno-Vega.

Correo electrónico:

maryl-morenov@unilibre.edu.co

Recibido: 06/05/2020

Aceptado:01/06/2020 
MATERIAL AND METHODS: observational, descriptive, retrospective, cross-sectional study included 523 medical records asthma exacerbation patients treated in the emergency room of a fourth-level healthcare center in Barranquilla between January and December 2017.

RESULTS: $0.93 \%$ all emergencies attended during 2017, represented to asthma exacerbation patients older than 16 years old. Exacerbation median was 1.43 visits per day. $47+/-19$ years patients mean age. $68 \%$ were women. $13.1 \%$ events occurred on September and associated triggering main factor was viral infection in $93.2 \%$.

CONCLUSIONS: this study provides data that highlights the important burden represents exacerbations asthma adult population in healthcare system, in relation to the attention provided at the emergency services, more frequently in rainy season, and suggests viral respiratory infections could be underlying cause for it.

Keywords: Asthma; Exacerbation; Clinical; Sociodemographic.

\section{Introducción}

El asma es uno de los grandes problemas de salud pública a nivel mundial, que afecta a personas de cualquier edad y cuya distribución es universal. La mayoría de los pacientes con asma permanecen sintomáticos a pesar del tratamiento de mantenimiento y experimentan exacerbaciones, las cuales son indicativas de un control deficiente del asma y pueden estar determinadas por la propia naturaleza de la enfermedad, pero sobre todo suelen deberse a factores exógenos, como infecciones respiratorias, contaminantes ambientales y un tratamiento inadecuado o falta de adherencia a este (1).

La prevalencia del asma ha aumentado más del $45 \%$ desde finales de los años setenta (2) y, aunque algunos países han visto una disminución en la hospitalización por asma, la carga global de las exacerbaciones ha aumentado casi un $30 \%$ en los últimos 20 años; de hecho, las exacerbaciones son la causa de morbilidad y mortalidad en pacientes con asma, lo que incrementa el costo anual del tratamiento hasta tres veces. Los diferentes estudios de prevalencia han puesto de manifiesto una importante tasa de subdiagnóstico del asma y una tendencia en aumento de la prevalencia si el diagnóstico se hace por autorreporte del paciente en comparación a cuando lo efectúa un médico (3).

Gran parte de los estudios están dirigidos hacia la caracterización del asma infantil, por lo que existe escasa literatura médica de estudios centrados en la población adulta y edad avanzada, en quienes se podría encontrar una mayor dificultad al momento del diagnóstico (por superposición de asma y enfermedad pulmonar obstructiva crónica [EPOC] o insuficiencia cardíaca congestiva) (4). No hay datos a nivel nacional respecto a la caracterización clínica y demográfica de la exacerbación asmática. El objetivo de este estudio es conocer la frecuencia de las exacerbaciones del asma en un servicio de urgencias de un centro de cuarto nivel, describir sus características clínicas y sociodemográficas, y determinar si existía algún factor de riesgo asociado con los episodios.

\section{Material y métodos}

Se realizó un análisis retrospectivo de los pacientes mayores de 16 años atendidos por exacerbaciones asmáticas en el servicio de urgencias de la Organización Clínica General del Norte, en Barranquilla, Atlántico, durante el período comprendido entre el 1 enero de 2017 y el 31 diciembre de 2017. Se realizó una búsqueda dirigida por fechas (1 de enero de 2017 hasta 31 diciembre de 2017) y con filtros de diagnóstico según CIE 10: J459 asma, J450 asma predominantemente alérgica, J451 asma no alérgica, J458 asma mixta, J46 estado asmático. Se recogió la información en un sistema de registro en el que se incluyó el diagnóstico, el nombre y los apellidos del paciente, el número de la historia clínica, la edad, el sexo, la dirección, la fecha de ingreso, la hora de ingreso y el destino del paciente en el alta del servicio de urgencias (domicilio $u$ hospitalización en piso/unidad de cuidados intensivos [UCI]). Asimismo, la distinción entre nuevos eventos (definidos como ingresos en un tiempo mayor de 15 
días del ingreso previo) y recaída temprana (definidos como ingresos en un tiempo menor de 15 días del ingreso previo).

La base de datos se elaboró tras una revisión sistemática de las historias del archivo de urgencias y, posteriormente, se analizaron las variables de acuerdo con su naturaleza: para las variables cualitativas se realizó un cálculo de las frecuencias absolutas y relativas, mientras que las variables cuantitativas se analizaron con medidas de tendencia central y dispersión, a través de software SPSS versión 22. Durante todas las etapas de la presente investigación se garantizó la confidencialidad de la información contenida en las historias clínicas, se aplicaron las pautas internacionales de la declaración de Helsinki, como propuesta de principios éticos para la investigación médica en seres humanos, incluida la investigación del material humano y de información identificables, y la normativa del Ministerio de Salud, en la Resolución 8430 de 1993.

\section{Resultados}

Durante el año 2017 fueron atendidos en el servicio de urgencias del centro de estudio 56090 ur- gencias, de las cuales 4941 fueron enfermedades del sistema respiratorio (el 8,8\% del total de las urgencias atendidas), dentro de las cuales se encontraron 523 registros $(0,93 \%$ del total de las urgencias atendidas) relacionadas con una exacerbación asmática en pacientes mayores de 16 años. Se discriminaron las recaídas tempranas (reingreso en menos de 15 días, correspondientes a 31 eventos) de los nuevos eventos (reingresos después de 15 días, correspondientes a 492 eventos). Las características generales se describen en la Tabla 1.

\section{Características sociodemográficas}

Un total de 354 pacientes ( $84 \%$ ) recibió atención en una sola ocasión, mientras que $67(15,9 \%)$ acudieron dos o más veces (2-6). Al observar en detalle las características sociodemográficas de los pacientes que ingresaron en una sola ocasión, se encontró que el $68 \%$ son mujeres y proceden de la zona urbana en un $96 \%$; de los 67 pacientes readmitidos, el $73 \%$ eran mujeres, y solo el $3 \%$ se encontraba en la zona rural. Al comparar los grupos, no se hallaron diferencias significativas $(p<0,05)$, a excepción del estado civil $(p=0,04)$. (Tabla 2).

Tabla 1. Características de las exacerbaciones de asma (Barranquilla, 2017)

\begin{tabular}{|lc|}
\hline \multicolumn{1}{|c|}{ Descripción general } & Resultados \\
\hline Número de exacerbaciones & 523 \\
\hline Número de pacientes & 421 \\
\hline Edad & $47+/-19$ años \\
\hline Sexo (mujeres) & $68 \%$ \\
\hline Exacerbaciones/día & $1,43(0-7)$ \\
\hline Número de recaída temprana & 31 \\
\hline Media de tiempo a recaída & $5,7+/-2,3$ días \\
Número de nuevos eventos & 492 \\
\hline Media de tiempo al nuevo evento & $80+/-57$ días \\
\hline
\end{tabular}


Tabla 2. Distribución porcentual de algunas características sociodemográficas de los pacientes con exacerbación de asma (Barranquilla, 2017)

\begin{tabular}{|c|c|c|c|c|c|c|}
\hline \multirow[t]{2}{*}{ Variable } & \multirow[t]{2}{*}{ Categoría } & \multicolumn{2}{|c|}{ Única admisión } & \multicolumn{2}{|c|}{ Readmitido } & \multirow[t]{2}{*}{$P$} \\
\hline & & $\mathbf{N}$ & $\%$ & $\mathbf{N}$ & $\%$ & \\
\hline \multirow[t]{2}{*}{ Sexo } & Mujer & 240 & 68 & 49 & 73 & \multirow{2}{*}{0,38} \\
\hline & Hombre & 114 & 32 & 18 & 27 & \\
\hline \multirow[t]{2}{*}{ Procedencia } & Urbano & 341 & 96 & 65 & 97 & \multirow{2}{*}{0,78} \\
\hline & Rural & 13 & 4 & 2 & 3 & \\
\hline \multirow[t]{6}{*}{ Estado civil } & Casado & 124 & 35 & 30 & 45 & \multirow{6}{*}{0,046} \\
\hline & Soltero & 139 & 39 & 14 & 21 & \\
\hline & Unión libre & 54 & 15 & 11 & 16 & \\
\hline & Viudo & 25 & 7 & 6 & 9 & \\
\hline & Divorciado & 7 & 2 & 3 & 5 & \\
\hline & Sin dato & 5 & 1 & 3 & 5 & \\
\hline \multirow[t]{10}{*}{ Ocupación } & Hogar & 105 & 30 & 18 & 27 & \\
\hline & Sin dato & 88 & 25 & 21 & 31 & \\
\hline & Educación & 59 & 17 & 9 & 13 & \\
\hline & Oficios varios & 38 & 11 & 7 & 10 & \\
\hline & Comercial & 15 & 4 & 2 & 3 & \\
\hline & Salud & 15 & 4 & 1 & 2 & \multirow{5}{*}{0,69} \\
\hline & Cesante & 14 & 4 & 6 & 9 & \\
\hline & Administrativo & 13 & 4 & 2 & 3 & \\
\hline & Abogado & 4 & 1 & 1 & 2 & \\
\hline & Cocina & 3 & 1 & 0 & 0 & \\
\hline \multirow[t]{3}{*}{ Aseguradora } & Contributivo & 221 & 62 & 41 & 61 & \multirow{3}{*}{0,91} \\
\hline & Especial & 114 & 32 & 23 & 34 & \\
\hline & Subsidiado & 19 & 5 & 3 & 5 & \\
\hline \multirow[t]{5}{*}{ Edad } & $>60$ & 108 & 31 & 26 & 39 & \multirow{5}{*}{0,43} \\
\hline & $50-59$ & 62 & 18 & 14 & 21 & \\
\hline & $35-49$ & 65 & 18 & 12 & 18 & \\
\hline & $18-34$ & 105 & 30 & 13 & 19 & \\
\hline & $<17$ & 14 & 4 & 2 & 3 & \\
\hline
\end{tabular}


El promedio de la edad de los pacientes con un solo ingreso fue de $47 \pm 19$ años, mientras que, en los pacientes readmitidos, fue de $52 \pm 20$ años (Tabla 2).

El índice de masa corporal (IMC) en los pacientes con exacerbación de asma que ingresaron en una sola ocasión fue superior al IMC de los readmitidos (27,1 \pm 5,1 frente a 24,8 \pm 8) (Tabla 3).

\section{Mes y hora de ingreso}

El mayor número de nuevos eventos se presentaron en el mes de septiembre, con el 13,1\% (74 eventos), y el menor número se dio en el mes de febrero, con el 4,3\% (20 eventos). Respecto a la recaída temprana, se encontró que el mayor número fue en marzo con el 22,6\% (7 eventos), y el menor número se dio en los meses de junio y octubre con igual representación porcentual en cada mes del 3,2 \% (1 evento). Se presentó un mayor número de eventos en horas de la tarde $(37,8 \%)$, seguido de las horas de la mañana, la noche y la madrugada con el $30,7 \%, 25 \%$ y el 5,7 \%, respectivamente. En cuanto las recaídas tempranas, se presentaron en igual proporción en horas de la tarde y la noche, con el $32,3 \%$ cada uno, seguido de los horarios de la mañana y la madrugada, con el $29 \%$ y el 6,5\% (Tabla 4).

\section{Síntomas y signos asociados}

Los síntomas más frecuentes referidos por los pacientes fueron tos y disnea en el 94,5\% y 93,9\%, respectivamente, tanto en los nuevos eventos como en las recaídas tempranas. Por otro lado, el hallazgo principal en el examen físico fue sibilancia en ambos grupos $(90 \%$ y $80 \%$, respectivamente); posteriormente, la disminución del murmullo vesicular en 26,4 \% y $41,9 \%$ (Tabla 5).

Tabla 3. Distribución porcentual de la variable IMC y subgrupo de obesidad en los pacientes de única admisión y los readmitidos (Barranquilla, 2017)

\begin{tabular}{|llcc|}
\hline \multicolumn{1}{c}{ Variable } & \multicolumn{1}{c}{ Categoría } & Única admisión & Readmitido \\
\cline { 3 - 4 } & & $\mathbf{n}(\%)$ & $\mathbf{n}(\%)$ \\
\hline IMC & Bajo peso & $0(0)$ & $1(2)$ \\
& Normal & $72(34)$ & $17(32)$ \\
$\mathrm{kg} / \mathrm{m}^{2}$ & Sobrepeso & $93(44)$ & $22(42)$ \\
& Obesidad & $48(23)$ & $13(25)$ \\
Total & & $213(100)$ & $53(100)$ \\
Subgrupos de obesidad & Obesidad grado I & $24(50)$ & $12(92)$ \\
& Obesidad grado II & $18(38)$ & $1(8)$ \\
& Obesidad mórbida & $6(13)$ & $0(0)$ \\
Total & & $48(100)$ & $13(100)$ \\
\hline
\end{tabular}

Tabla 4. Distribución porcentual de los ingresos y los reingresos de los pacientes con exacerbación de asma según la jornada (Barranquilla, 2017)

\begin{tabular}{|lcc|}
\hline \multicolumn{1}{|c}{ Jornada } & Nuevo evento & Recaída \\
\cline { 2 - 3 } & $\mathbf{n}(\%)$ & $\mathbf{n}(\%)$ \\
\hline Mañana (06:00 a. m. a 12:00 a. m.) & $151(30,7)$ & $9(29)$ \\
\hline Tarde (12:00 a. m. a 18:00 p. m.) & $186(37,8)$ & $10(32,3)$ \\
\hline Noche (18:00 p. m. a 0:00 a. m.) & $127(25,8)$ & $10(32,3)$ \\
\hline Madrugada (0:00 a. m. a 6:00 a. m.) & $28(5,7)$ & $2(6,5)$ \\
\hline
\end{tabular}


Tabla 5. Síntomas y signos predominantes en los pacientes con exacerbación de asma (Barranquilla, 2017)

\begin{tabular}{|c|c|c|c|c|}
\hline Variable & Nuevo evento n (492) & $\%$ & Recaída temprana n (31) & $\%$ \\
\hline \multicolumn{5}{|l|}{ Síntomas } \\
\hline Tos & 465 & 94,5 & 31 & 100 \\
\hline Disnea & 462 & 93,9 & 30 & 96,8 \\
\hline Dolor torácico & 28 & 5,7 & 4 & 12,9 \\
\hline Síntomas generales & 153 & 31,1 & 11 & 35,5 \\
\hline \multicolumn{5}{|l|}{ Signos } \\
\hline Sibilancias & 443 & 90,0 & 25 & 80,6 \\
\hline DMV & 130 & 26,4 & 13 & 41,9 \\
\hline Roncus & 81 & 16,5 & 4 & 12,9 \\
\hline Tirajes & 32 & 6,5 & 2 & 6,5 \\
\hline Taquipnea & 10 & 2,0 & 0 & 0 \\
\hline Normal & 7 & 1,4 & 3 & 9,7 \\
\hline
\end{tabular}

DMV: disminución del murmullo vesicular.

\section{Paraclínicos}

En 71 casos se realizó algún paraclínico (14,4 \%), donde el $49 \%$ correspondió a un hemograma y el $99,2 \%$ a la realización de una radiografía de tórax (66 casos); al observar en detalle, el 57,1 \% fueron normales, en el 28,5\% se halló leucocitosis $>11500$ y en el $14,2 \%$ eosinofilia (>400 eosinófilos). Con respecto a los hallazgos en la radiografía de tórax, se encontraron en $65,1 \%, 28,7 \%$ y $6,06 \%$, normales, reforzamiento peribronquial y atrapamiento aéreo, respectivamente (Figuras 1 y 2).

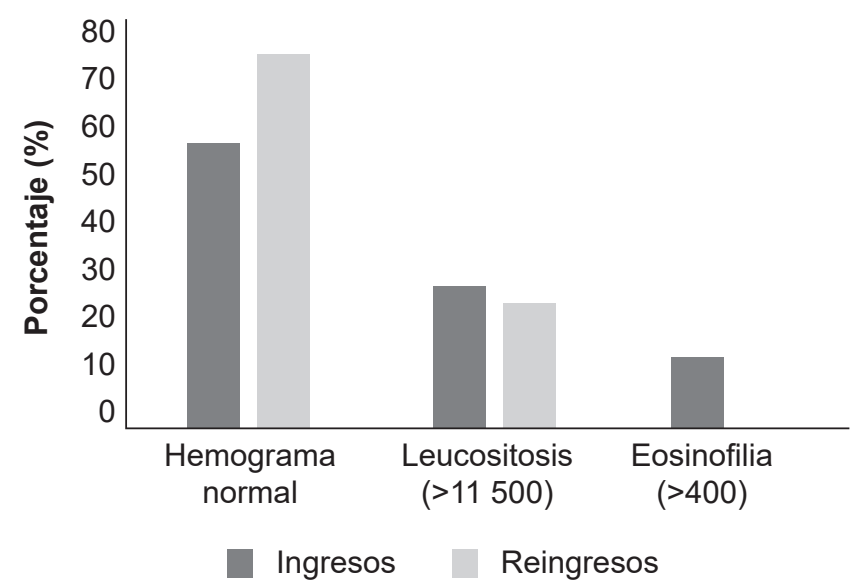

Figura 1. Distribución porcentual de pacientes con exacerbación de asma, a los que se les realizaron exámenes paraclínicos (Barranquilla, 2017).

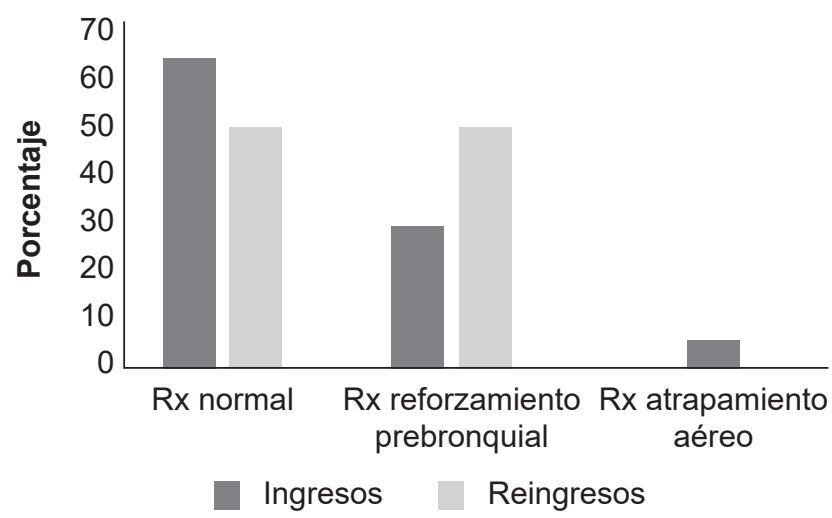

Figura 2. Distribución porcentual de pacientes con exacerbación de asma, a los que se les realizó radiografía de tórax (Barranquilla, 2017).

\section{Conducta de egreso}

Del total de pacientes con un solo ingreso, 487 casos $(98,9 \%)$ egresaron del centro de salud y $5(1,01 \%)$ permanecieron en observación. Del total de reingresos, 29 casos egresaron $(93,5 \%)$ y 2 casos $(6,4 \%)$ continuaron en observación.

\section{Factores detonantes}

Del total de pacientes con un nuevo evento en el período evaluado se encontró que en 117 casos $(23,4 \%)$ se identificó un factor detonante y, de esos casos, 109 $(93,2 \%)$ correspondían a una infección viral y $8(6,8 \%)$ 
se relacionaron con la exposición a tóxicos como pinturas, desinfectantes, entre otros. En los pacientes con recaída temprana, 11 casos registraron un factor detonante y el $100 \%$ de ellos correspondían a una infección viral.

\section{Discusión}

El reconocimiento de la carga hospitalaria por exacerbación de asma es un método indirecto para estimar el impacto de las enfermedades obstructivas de la vía aérea en la población y para medir la calidad de las estrategias de tratamiento de estas enfermedades (5). En Colombia no existen muchos estudios acerca de la frecuencia hospitalaria de los pacientes con exacerbación asmática y, además, gran parte de los estudios están dirigidos hacia la caracterización del asma infantil, dejando escasa literatura médica de estudios centrados en la población adulta y edad avanzada, con evidencia de una alta prevalencia de la enfermedad que va en aumento (4-7). Este hecho se asocia con un incremento importante en el número de consultas a los servicios de urgencias por exacerbaciones asmáticas; por esta razón, investigaciones como esta permiten iniciar un reconocimiento más real de la magnitud del problema en el país.

Se ha encontrado que hasta $8,8 \%$ del total de las urgencias atendidas durante el año 2017 fueron motivadas por enfermedades del sistema respiratorio (segundo diagnóstico más frecuente después del traumatismo), dentro de las cuales se estimó una frecuencia de $0,93 \%$ relacionadas con una exacerbación asmática en pacientes mayores de 16 años, igual a la reportada por Otero y colaboradores, donde se analizó un grupo de edad similar (8). Este porcentaje podría ser aún mayor teniendo en cuenta que no se incluyó la población pediátrica como se describe $(9,10)$.

La edad media de los pacientes con exacerbación asmática fue mayor que la referida por otros autores, como Rea y colaboradores (11), quienes observaron una edad media de 29,9 años, y Suruki y colaboradores, con una media de 38 años (en la cohorte de Estados Unidos) (9). Los dos factores que pueden haber influido en la elevada media de la edad que se observó corresponden al hecho de no incluir a la población in- fantil y no establecer un límite de edad superior a la hora de incluir pacientes, tal y como lo hacen Rea y colaboradores $(11,12)$, quienes no incluyeron a pacientes con una edad superior a 55 años, lo cual se corresponde con trabajos con criterios de inclusión similares (8).

Se observó que más del $50 \%$ de los pacientes tienen más de 50 años, lo cual incluye un sector de la población cada vez más numeroso, debido al envejecimiento de la población, y en el que el asma parece tener una prevalencia no inferior a la observada en la población de mediana edad (7-9\%) (13). El resto de la población está conformada por pacientes entre la segunda y la quinta década de la vida, lo que corresponde al grupo con mayor vida productiva.

Al igual que en otras series publicadas $(8,9,14$, 15), se observó un claro predominio del sexo femenino en los adultos que consultan a urgencias por exacerbación asmática.

La media diaria de casos de exacerbación de asma observada fue significativamente superior en los meses de lluvia como mayo, septiembre y octubre (16), lo cual se ha descrito en otros estudios, donde esto se ha relacionado con factores como infecciones respiratorias de origen viral y variables meteorológicas; sin embargo, la mayor tasa de reingresos se dio en el mes de marzo, que corresponde a un período seco, lo que podría reflejar la participación de otras variables en la determinación de la exacerbación $(8,17)$.

La distribución horaria de las exacerbaciones asmáticas no sigue el clásico patrón circadiano que han reflejado algunos estudios, en los que se describe el empeoramiento del asma en horas nocturnas, como consecuencia de las variaciones que experimentan las vías aéreas, las cuales alcanzan un calibre mínimo a las 4 horas (18); esto sumado al incremento del tono vagal, que favorece la broncoconstricción nocturna (19). En la distribución de los eventos se encontraron resultados similares a los descritos en otros estudios $(8,20,21)$, donde menos de la cuarta parte de los pacientes acudieron al servicio de urgencias durante la madrugada. La menor afluencia nocturna se podría explicar por el mejor conocimiento de la fisiopatología de la enfermedad y el uso de inhaladores para el control de la crisis asmática. 
Más de la mitad de los pacientes con exacerbación de asma, en los cuales se registró el IMC, presentaban un valor por encima del normal, y al análisis por subgrupo la mayoría correspondía a obesidad grado I, lo que se correlaciona con lo descrito en otros estudios $(22,23)$. El estudio TENOR mostró que la proporción de pacientes con un IMC $>30$ fue superior en los pacientes que experimentaron exacerbaciones graves del asma dentro de los 1,5 años anteriores, que las que no experimentó (24); resultados contrarios reportaron Tanaka y colaboradores, lo cual atribuyeron a la diferencia racial (25). Se recomiendan estudios con un diseño prospectivo para investigar la asociación sustancial entre obesidad y exacerbaciones en el asma en Colombia.

Los síntomas iniciales más comunes corresponden a tos seca y disnea, representados con $94,5 \%$ y $93,9 \%$, respectivamente, mientras que la sibilancia fue el signo más frecuente, seguido de la disminución del murmullo vesicular en $90 \%$ y $26,4 \%$, respectivamente. Resultados similares encontraron Pola y colaboradores (26).

Como factores predictores de las exacerbaciones y la hospitalización, se ha descrito la presencia significativa de exacerbaciones y hospitalizaciones en el año previo $(9,24,26)$ así como eosinofilia en la sangre o el esputo (27). En el presente estudio no se pudo contar con el registro de exacerbaciones previas, y solo al $7 \%$ de la población se le realizó un hemograma; de estos, solo en el $14 \%$ se documentó eosinofilia, la cual no correspondió a los pacientes en quienes se consideró observación. Durante el seguimiento durante un año de las cohortes analizadas, Suruki y colaboradores documentaron que los pacientes con $\geq 2$ exacerbaciones presentaban una enfermedad más grave (con un escalón más alto en la escala terapéutica de GINA), así como un historial de exacerbaciones más frecuentes y una clasificación de asma grave no controlada (9). Este estudio permitió establecer que hasta el 15,9\% de la población analizada presentó más de un ingreso durante el año (media 2,4 $\pm 0,8$, con una mediana de tiempo a la consulta de 91 días), lo que podría tener relación con una enfermedad más grave y un menor control de esta.

Varios factores pueden contribuir a la exacerbación asmática, incluyendo infecciones, el uso insuficiente de medicamentos para el control de esta o la exposición a alérgenos o contaminantes (28). Del total de eventos de la presente serie, se documentó un factor detonante en el $23,4 \%$, correspondiente a una infección respiratoria $(93,2 \%)$ similar a los resultados obtenidos en otras series $(8,12,25,29)$, que se considera importante porque contribuye hasta en el $90 \%$ de las exacerbaciones, especialmente en los picos estacionales que coinciden con las temporadas frías y meses de lluvia (30).

El papel de la infección en el asma varía desde la contribución al desarrollo clínico inicial de la enfermedad hasta como factor precipitante de las exacerbaciones. Al respecto, se ha reunido evidencia creciente que implica principalmente a los virus (rinovirus y virus sincitial respiratorio) y las infecciones bacterianas atípicas como Mycoplasma pneumoniae y Chlamydia pneumoniae u hongos en el caso de la aspergilosis broncopulmonar alérgica (31). No obstante, es necesario tener presente que la estimación de los casos de infección viral se realiza según los criterios clínicos, ya que no hubo estudios de determinación de la reacción en cadena de la polimerasa (PCR) viral u otras técnicas de aislamiento para recuperar los gérmenes causantes del proceso respiratorio; por tanto, la única conclusión que puede establecerse es la confirmación de la existencia de una relación entre las infecciones respiratorias y las exacerbaciones de asma.

A pesar de la utilidad de la clínica, se recomienda que las decisiones terapéuticas se sustenten, además, en la evaluación de medidas repetidas del pico de flujo espiratorio (PFE) o el volumen espiratorio forzado en 1 segundo $\left(\mathrm{VEF}_{1}\right)$, junto con la monitorización continua de la saturación de oxígeno, para valorar adecuadamente la gravedad de la obstrucción, el intercambio gaseoso y la respuesta al tratamiento; de hecho, las medidas espirométricas pueden ayudar a identificar al paciente con un mayor riesgo de exacerbación (32, 33). Por tanto, llama la atención en los resultados obtenidos el uso nulo del PFE en los eventos como una herramienta para guiar la decisión del tratamiento y el destino del paciente en función de la hospitalización o el egreso, que a la postre impacta en el seguimiento apropiado del paciente.

A la vista de estos resultados, se reconoce, al menos a nivel local, que la agudización del asma es un motivo frecuente de consulta en los servicios de urgencias hos- 
pitalarias, lugar al que acude una proporción elevada de pacientes de modo reiterativo y, en mayor proporción, en la temporada invernal.

Favorece la evidencia histórica de la relación positiva entre el género femenino, las infecciones respiratorias y las exacerbaciones de asma, así como sugiere la probable relación entre obesidad y exacerbación; sin embargo, se recomiendan, frente a este último, estudios con diseño prospectivo para investigarlo apropiadamente.

Estos resultados sugieren que, considerando el tratamiento de base del paciente y la frecuencia de uso, así como el número de exacerbaciones durante el año anterior, se puede definir el control del asma y predecir el riesgo de exacerbaciones futuras.

Finalmente, se demuestra el nulo uso del PEF como herramienta para guiar la decisión del tratamiento y el destino del paciente.

Este estudio tiene ciertas limitaciones comunes a los estudios retrospectivos en general, como que algunas variables de los pacientes no pudieron determinarse en todos los casos. Además, los datos provinieron de un único centro, por lo que los resultados pueden no reflejar adecuadamente la situación en otros centros médicos. El diagnóstico de asma se basó en el registrado al ingreso de la historia clínica elaborada por el médico que trató al paciente; sin embargo, no se realizaron pruebas complementarias adicionales para confirmar el diagnóstico de asma, lo que significa que algún diagnóstico erróneo es posible.

\section{Recomendación}

Con base en los resultados obtenidos en este estudio se propone enfocar los planes de educación no solo en el paciente y el familiar asmático ya captado, sino en acudir a las instituciones de salud e iniciar una capacitación para los médicos que tengan el primer contacto en este tema. Además, redireccionar el proceso educativo hacia el uso del flujómetro como mecanismo de autocontrol en el asma, fomentar la adherencia al tratamiento de sostén y usar apropiadamente la terapéutica de rescate.

\section{Conflicto de interés}

Los autores de este artículo declaran no tener ningún conflicto de interés.

\section{Referencias}

1. Drazen J. Asma. En: Goldman L, Schafer A (editores). Tratado de Medicina Interna. Madrid: Elsevier; 2017. p. 548-555.

2. Lugogo N, Que L, Gilstrap D, Kraft M. Asthma: Clinical Diagnosis and Management. En: Murray J, Nadel J, Broaddus C, Mason R. Textbook of respiratory medicine. España: Elsevier; 2016. p. 731-750.

3. Global strategy for asthma management and prevention 2020 update. Global initiative for asthma (GINA) [consultado el 15 de abril de 2020]. Disponible en: https:/ginasthma.org/ wp-content/uploads/2020/06/GINA-2020-report_20_06_041-wms.pdf

4. Holgate S. Asthma Pathogenesis. En: Adkinson F, Bochner B, Burks W, Busse W. Middleton's Allergy: Principles and Practice. España: Elsevier; 2014. p. 812-841.

5. Nowak RM, Hurd SS, Skobeloff EM, Taggart VS. Asthma research: future directions for emergency medicine. Ann Emerg Med. 1996;27:244-9. doi: 10.1016/s0196-0644(96)70331-1

6. Ocampo J, Gaviria R, Sánchez J. Prevalencia del asma en América Latina. Mirada crítica a partir del ISAAC y otros estudios. Rev Alerg Mex. 2017;64:188-197.

7. González-García M, Caballero A, Jaramillo C, Maldonado D, Torres-Duque CA. Prevalence, risk factors and underdiagnosis of asthma and wheezing in adults 40 years and older: A population-based study. J Asthma. 2015;52(8):823-30. doi: 10.3109/02770903.2015.1010733

8. Otero I, Blanco M, Montero C, Valiño P, Verea H. Características epidemiológicas de las exacerbaciones por EPOC y asma en un hospital general. Arch Bronconeumol. 2002;38:256-62.

9. Suruki RY, Daugherty JB, Boudiaf N, Albers FC. The frequency of asthma exacerbations and healthcare utilization in patients with asthma from the UK and USA. BMC Pulm Med. 2017;17(1):74. doi: 10.1186/s12890-017-0409-3

10. Herrera A, Escobar A, Saavedra M, Salgado E, Palavecino T. Exacerbación de asma infantil en unidad de paciente crítico: caracterización clínica. Revista chilena de enfermedades respiratorias. 2011;27(1):26-30.

11. Rea HH, Garrett JE, Mulder J, Chapman KR, White JG, Rebuck AS. Emergency room care of asthmatics: a comparison between Auckland and Toronto. Ann Allergy. 1991;66(1):48-52.

12. Gonzalez-Barcala FJ, Calvo-Alvarez U, Garcia-Sanz MT, Bourdin A, Pose-Reino A, Carreira JM, Moure-Gonzalez JD, Garcia-Couceiro N, Valdes-Cuadrado L, Muñoz X. Characteristics and prognosis of near-fatal asthma exacerbations. Am J Med Sci. 2015;350(2):98-102. doi: 10.1097/ MAJ.0000000000000518 
13. Gil PR. Epidemiología del asma en Colombia. Asociación Colombiana de Neumología y cirugía de tórax. Revista Colombiana de Neumología. 2015;27(3):223-5.

14. Skobeloff EM, Spivey WH, St Clair SS, Schoffstall JM. The influence of age and sex on asthma admissions. JAMA. 1992;268(24):3437-40.

15. Singh AK, Cydulka RK, Stahmer SA, Woodruff PG, Camargo CA Jr. Sex differences among adults presenting to the emergency department with acute asthma. Multicenter Asthma Research Collaboration Investigators. Arch Intern Med. 1999 ;159(11):1237-43. doi: 10.1001/archinte.159.11.1237

16. Boletín climatológico mensual. [Internet]. Colombia: IDEAM - Instituto de Hidrología, Meteorología y Estudios Ambientales; 2017. [Citado 10 de mayo 2020]. Disponible en http://www.ideam.gov.co/web/tiempo-y-clima/climatologico-mensual/-/document_library_display/xYvlPc4uxk1Y/ view/18512937

17. Castro CR, Tarabichi Y, Gunzler DD, Ayache M. Seasonal trends in asthma exacerbations: Are they the same in asthma subgroups? Ann Allergy Asthma Immunol. 2019;123(2):220222. doi: 10.1016/j.anai.2019.05.001

18. Buff DD, Bavli SZ, Bloch MH, Serebryansky BM, Aman CS. Circadian patterns of emergency asthma presentations: implications for staffing and treatment. Respir Care. 1995;40(6):624-30.

19. Barnes PJ. Inflammatory mechanisms and nocturnal asthma. Am J Med. 1988;85(1B):64-70. doi: 10.1016/00029343(88)90245-8

20. Karras DJ, D'Alonzo GE, Heilpern KL. Is circadian variation in asthma severity relevant in the emergency department? Ann Emerg Med. 1995;26(5):558-62. doi: 10.1016/s01960644(95)70004-8

21. Brenner BE, Chavda KK, Karakurum MB, Karras DJ, Camargo CA Jr; MARC Investigators. Circadian differences among 4,096 emergency department patients with acute asthma. Crit Care Med. 2001;29(6):1124-9. doi: 10.1097/00003246200106000-00005

22. Dixon AE, Holguin F, Sood A, Salome CM, Pratley RE, Beuther DA, et al. An official American Thoracic Society Workshop report: obesity and asthma. Proc Am Thorac Soc. 2010;7(5):325-35. doi: 10.1513/pats.200903-013ST

23. Dixon AE, Pratley RE, Forgione PM, Kaminsky DA, Whittaker-Leclair LA, Griffes LA, et al. Effects of obesity and bariatric surgery on airway hyperresponsiveness, asthma control, and inflammation. J Allergy Clin Immunol. 2011;128(3):508-15.e1-2. doi: 10.1016/j.jaci.2011.06.009

24. Miller MK, Lee JH, Miller DP, Wenzel SE; TENOR Study Group. Recent asthma exacerbations: a key predictor of future exacerbations. Respir Med. 2007;101(3):481-9. doi: 10.1016/j.rmed.2006.07.005

25. Tanaka A, Uno T, Sato H, Jinno M, Hirai K, Miyata Y, et al. Predicting future risk of exacerbations in Japanese patients with adult asthma: A prospective 1-year follow up study. Allergol Int. 2017;66(4):568-573. doi: 10.1016/j.alit.2017.02.013

26. Pola-Bibian B et al. Asthma exacerbations in a tertiary hospital: clinical features, triggers, and risk factors for hospitalization. Journal Investig Allergol Clin Immunol. 2017; 27: 238-245. Pola-Bibian B, Dominguez-Ortega J, Vilà-Nadal G, Entrala A, González-Cavero L, Barranco P, et al. Asthma exacerbations in a tertiary hospital: clinical features, triggers, and risk factors for hospitalization. J Investig Allergol Clin Immunol. 2016:0. doi: 10.18176/jiaci.0128

27. Price DB, Rigazio A, Campbell JD, Bleecker ER, Corrigan CJ, Thomas M, et al. Blood eosinophil count and prospective annual asthma disease burden: a UK cohort study. Lancet Respir Med. 2015;3(11):849-58. doi: 10.1016/S22132600(15)00367-7

28. National Asthma Education and Prevention Program. Expert Panel Report 3 (EPR-3): Guidelines for the Diagnosis and Management of Asthma-Summary Report 2007. J Allergy Clin Immunol. 2007;120(5 Suppl):S94-138. doi: 10.1016/j. jaci.2007.09.043

29. Teichtahl H, Buckmaster N, Pertnikovs E. The incidence of respiratory tract infection in adults requiring hospitalization for asthma. Chest. 1997;112(3):591-6. doi: 10.1378/ chest.112.3.591

30. Weiss KB. Seasonal trends in US asthma hospitalizations and mortality. JAMA. 1990;263(17):2323-8.

31. Darveaux JI, Lemanske RF Jr. Infection-related asthma. J Allergy Clin Immunol Pract. 2014;2(6):658-63. doi: 10.1016/j.jaip.2014.09.011

32. Quezada W, Kwak ES, Reibman J, Rogers L, Mastronarde J, Teague WG, et al. Predictors of asthma exacerbation among patients with poorly controlled asthma despite inhaled corticosteroid treatment. Ann Allergy Asthma Immunol. 2016;116(2):112-7. doi: 10.1016/j.anai.2015.11.011

33. Rodrigo C. Asma aguda severa: su manejo en la emergencia y cuidado intensivo. Medicina intensiva. 2006;30(9):460-70. 